were no significant differences between groups in terms of gender, age, and IBD diagnosis.

Reasons for stopping azathioprine were drug-related symptoms (33\% in LDG v 24\% in TDG), abnormal liver function tests $(8 \%$ in LDG $\mathrm{v} 3 \%$ in TDG), and pancreatitis (3\% in LDG $\mathrm{v} 3 \%$ in TDG). Significant leucopenic episodes (defined as white cell count $<3.5 \times 10^{9} / \mathrm{l}$ ) occurred in 2 LDG patients and 1 TDG patient. In the TDG patient this was transient and resolved before any dose adjustment was required.

In TDG patients, thiopurine metabolites were checked at week 4 and showed median thioguanine nucleotides level = $379 \mathrm{pmol} / 8 \times 10^{8}$ cells (range $176-520$ ), and median methylmercaptopurine $(\mathrm{MMP})$ level $=2438 \mathrm{pmol} / 8 \times 10^{8}$ cells (range 794 - 7277). Four patients in this group had their dose adjusted due to high MMP levels.

Conclusions Starting Azathioprine at a therapeutic $2 \mathrm{mg} / \mathrm{kg}$ dose, in IBD patients with normal TPMT level, appears to be as safe and well tolerated compared to using a low dose approach with gradual increase. This more direct approach has clear benefits in terms of achieving therapeutic response earlier, reducing the number of patient contacts, and saving IBD nurse or pharmacist time.

\section{P364 THE IBD BIOLOGICS MDT: IMPROVING PATIENT SAFETY, GOVERNANCE, WAITING TIMES AND EFFICIENCY SAVINGS}

Christopher Kelly, Nicole Haggarty, Ann Muir, Gillian Richardson, Pauline Morrison, lain Morrison, Laura Clark, Stuart Paterson, Santoshkumar Salunke*. NHS Forth Valley, Larbert, UK

\subsection{6/gutjnl-2020-bsgcampus.438}

Introduction Biologic therapies have an ever-increasing evidence base to support their use in treatment of inflammatory bowel disease (IBD). Increasing number of patients on such treatments coupled with reduction in outpatient capacity coupled with availability of newer and multiple biologic drugs can often lead to challenges in decision making regarding best biologic and difficulty in safely monitoring this effective, but potentially toxic therapy. It was hypothesized that a Biologic Multi-Disciplinary Team (MDT) meeting could make dramatic improvements in quality of care, improve safety and establish governance around use of biologics in our district general hospital. The project was carried out in partnership with Royal College of Physicians, London, IBD Quality Improvement (QI) collaborative.

\section{Treatment Optimisations} $60 / 113(53 \%)$

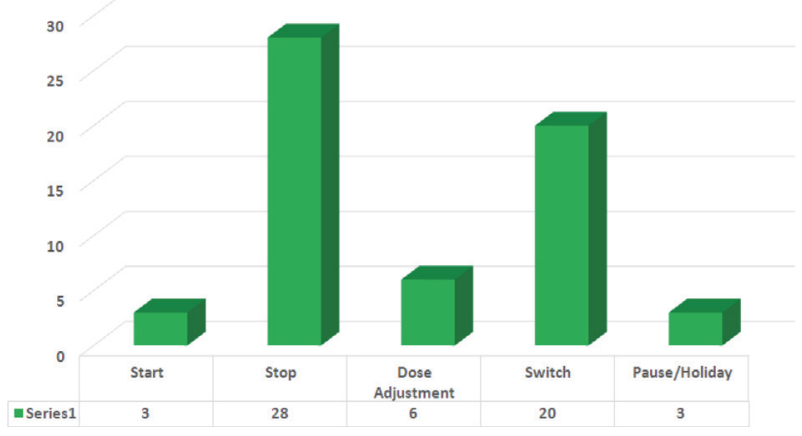

Abstract P364 Figure 1
Methods IBD biologics MDT was initiated along the principles of QI. The aims, drivers and personnel were agreed via driver diagram. The criteria to include patients in the MDT were agreed- Longest wait (Patients who were furthest away from their last clinical review), Clinical Need or Complex Biosimilar switch. The MDT met for 90 minutes twice monthly. At least 3 Consultants were required to make quorate decisions. Outcomes were agreed and recorded, a copy of which would be sent to the GP and the patient. The data was recorded on a new biologics database. The process underwent 'PDSA cycles'. Patient feedback was received.

Results Over the 1st 12 months, of the initial 225 patients on biologics (n) 113 (50\%) were discussed (patients on biologics rose to 284 , an increase of $26 \%$, during this period). Four different biologics were being used in these patients (Biosimilar infliximab, originator or biosimilar Adalimumab, Vedolizumab and Ustekinumab).The longest waiting time between clinical review reduced from 80 to 31 weeks. The MDT altered management in $60(53 \%)$ of patients It also led to further investigations in 49 (43\%). Drug cost savings of $£ 72206$ was noted. $74 \%$ of patients surveyed were satisfied with MDT discussion, with the remaining $26 \%$ indifferent to discussion.

Conclusions The Biologic MDT can provide a safe and effective framework for IBD patients on biologic agents.

\section{P365 GASTROENTEROLOGY OUTPATIENT TRANSFORMATION - CLINICAL ASSESSMENT SERVICE (CAS)}

Muhammad Taha Khan*, Sunil Sonwalkar, Ashwin Verma. Calderdale And Huddersfield Nhs Trust, Huddersfield, UK

\subsection{6/gutjnl-2020-bsgcampus.439}

Introduction Increasing referrals lead to prolonged waiting time for patients to be seen in the clinic, creating pressure to comply with national guidance on referral to treatment time and raised appointment slot issue.

Method Clinical assessment service clinic was introduced to assess and manage patients remotely without face to face appointments.

Currently we are running 2 Virtual Gastroenterology clinics per week, where 40 patients are reviewed.

New referral in CAS clinic results in the following 5 outcomes; inappropriate referral, face to face appointment, appointment with/after results, review remotely after investigations, advice to GP and discharge.

Results 1254 patients have been reviewed in CAS clinic in 9 months. $21.1 \%$ of those patients were discharged with advice to GP on first assessment of the referral, including inappropriate referrals. Out of remaining 937 patients, $64.7 \%$ went directly for investigations and 113 patients were discharged after investigations, following a remote review.

Conclusion CAS clinic faced challenges initially, such as familiarizing GPs and staff to the system. This was dealt with, by reaching out to them, conducting awareness seminars and teaching sessions run by the Consultants. CCG and administration were supportive.

DNA (did not attend) rate fell to $0 \%$ and the process helped save new and follow up appointment slots. The Trust gained a financial benefit of $£ 117,259$ in 9 months. 